\title{
Article
}

\section{Inflammatory Biomarker Score Identifies Patients with Six-Fold Increased Risk of One-Year Mortality after Pancreatic Cancer}

\author{
Alisa D. Kjaergaard ${ }^{1, *(D)}$, Inna M. Chen ${ }^{2}$, Astrid Z. Johansen ${ }^{2}$, Børge G. Nordestgaard ${ }^{3,4}$, Stig E. Bojesen ${ }^{3,4}$ \\ and Julia S. Johansen 2,4 \\ 1 Steno Diabetes Center Aarhus, Aarhus University Hospital, 8200 Aarhus, Denmark \\ 2 Department of Oncology, Herlev and Gentofte Hospital, Copenhagen University Hospital, \\ 2730 Herlev, Denmark; inna.chen@regionh.dk (I.M.C.); astrid.zedlitz.johansen@regionh.dk (A.Z.J.); \\ julia.sidenius.johansen@regionh.dk (J.S.J.) \\ 3 Department of Clinical Biochemistry, Herlev and Gentofte Hospital, Copenhagen University Hospital, \\ 2730 Herlev, Denmark; boerge.nordestgaard@regionh.dk (B.G.N.); stig.egil.bojesen@regionh.dk (S.E.B.) \\ 4 Department of Clinical Medicine, Faculty of Health and Medical Sciences, University of Copenhagen, \\ 2200 Copenhagen, Denmark \\ * Correspondence: alisa.kjaergaard@auh.rm.dk
}

check for updates

Citation: Kjaergaard, A.D.; Chen, I.M.; Johansen, A.Z.; Nordestgaard, B.G.; Bojesen, S.E.; Johansen, J.S. Inflammatory Biomarker Score Identifies Patients with Six-Fold Increased Risk of One-Year Mortality after Pancreatic Cancer. Cancers 2021, 13, 4599. https://doi.org/10.3390/ cancers13184599

Academic Editor: Ramzi M. Mohammad

Received: 12 August 2021 Accepted: 10 September 2021 Published: 13 September 2021

Publisher's Note: MDPI stays neutral with regard to jurisdictional claims in published maps and institutional affiliations.

Copyright: (c) 2021 by the authors. Licensee MDPI, Basel, Switzerland. This article is an open access article distributed under the terms and conditions of the Creative Commons Attribution (CC BY) license (https:// creativecommons.org/licenses/by/ $4.0 /)$.
Simple Summary: For 20 years, the CA 19-9 blood test has been the only broadly used biomarker of pancreatic ductal adenocarcinoma (PDAC). We lack easily available biomarkers to help differentiate patients between good, intermediate and poor survivors at the time of PDAC diagnosis. Using one of the largest studies of patients with PDAC, we found that a simple combination of blood tests, namely CRP, CA 19-9 and IL-6, into a single biomarker score was a better marker of oneyear survival than the currently recommended CA 19-9 alone or any other combination of the four inflammatory biomarkers examined (CRP, CA 19-9, IL-6 and YKL-40). However, since this is the first study examining this inflammatory biomarker score, future validation studies are needed. Moreover, CRP outperformed CA 19-9 in the majority of patients, thus questioning the routine use of CA 19-9 in patients with PDAC.

Abstract: We examined whether elevated plasma C-reactive protein (CRP), carbohydrate antigen (CA) 19-9, interleukin-6 (IL-6) and YKL-40, individually or combined, can identify poor survivors among patients with pancreatic ductal adenocarcinoma (PDAC). We measured CRP, CA 19-9, IL-6 and YKL-40 in 993 patients at the time of PDAC diagnosis. The biomarker score was the sum of biomarker categories, coded 0, 1 and 2 for low, intermediate and high plasma concentrations, respectively. High vs. low levels of CRP, CA 19-9 and IL-6 were each independently associated with a two-fold increased risk of one-year mortality. CRP performed best in patients with advanced and CA 19-9 in patients with low cancer stages. YKL-40 was not associated with mortality and, therefore, was not included in the biomarker score. Compared to the biomarker score $=0$, the multifactorially adjusted hazard ratios for one-year mortality were 1.56 (95\% confidence interval: $0.99-2.44)$ for score $=1,2.22(1.41-3.49)$ for score $=2,3.44(2.20-5.38)$ for score $=3,5.13(3.21-8.17)$ for score $=4$ and $6.32(3.84-10.41)$ for score $=5-6\left(p\right.$-value for trend $\left.=3 \times 10^{-31}\right)$. This score performed better than any single biomarker or combination of biomarkers when examined in similarly sized or other categories. In conclusion, a combination score of elevated CRP, CA 19-9 and IL-6 identified patients with six-fold higher one-year mortality.

Keywords: C-reactive protein; CA-19-9 antigen; interleukin-6; chitinase-3-like protein 1; carcinoma; pancreatic ductal

\section{Introduction}

Pancreatic cancer is currently the fourth and is projected to become the second leading cause of cancer deaths in both the United States of America and Europe (https:/ / gco.iarc.fr, 
accessed on 2 August 2021) [1,2]. Global incidence and mortality rates are slightly higher in men than in women, and historical incidence trends coincide with rising prevalences of smoking, overweight and diabetes [1,3]. There are usually no or few symptoms at early stages, and pancreatic cancer is therefore often diagnosed at a late stage [4]. Hence, the chances for complete resection and curative treatment are slim, and the overall five-year survival is only $9 \%$, with little improvements over the last years [1]. Importantly, we lack easily available biomarkers to help differentiate patients between good, intermediate and poor survivors at the time of diagnosis of pancreatic cancer.

Inflammation plays an important role in the development and progression of pancreatic cancer [5]. Numerous previous studies reported that inflammatory biomarkers, including C-reactive protein (CRP) and interleukin-6 (IL-6), were independently associated with overall survival in (from only 7 to 474 ) patients with pancreatic cancer [6-11]. Previous results from the Danish BIOPAC (BIOmarkers in patients with PAncreatic Cancer) study on 559-592 patients with pancreatic cancer found that plasma CRP, carbohydrate antigen 19-9 (CA 19-9), IL-6 and YKL-40 (see Supplementary Introduction) may each be independent biomarkers of overall survival $[12,13]$. However, for more than 20 years, CA 19-9 has remained the only broadly used biomarker of pancreatic ductal adenocarcinoma (PDAC) [14]. A recent Finnish study of 212 patients who were operated on for PDAC showed that a combination of elevated CRP $(\geq 3 \mathrm{mg} / \mathrm{L})$ and CA 19-9 $(>37 \mathrm{kU} / \mathrm{L})$ was associated with a three- to four-fold increased risk of mortality [15]. Furthermore, this combination of elevated CRP and CA 19-9 outperformed combinations of elevated CRP and decreased albumin (original and modified Glasgow prognostic scores) as well as other routine biochemical analyses (leukocytes, platelets, bilirubin and carcinoembryonic antigen) for prognostic purposes [15].

We therefore tested the hypothesis that elevated plasma CRP, CA 19-9, IL-6 and YKL40 individually and as a combined biomarker score are associated with poor survival in patients with PDAC.

\section{Materials and Methods}

This study adheres to the REMARK (Reporting Recommendations for Tumor Marker Prognostic Studies) guidelines.

We included 993 adult patients with histologically verified PDAC from the Danish BIOPAC ("BIOmarkers in patients with PAncreatic Cancer (BIOPAC) — can they provide new information of the disease and improve diagnosis and prognosis of the patients"; ClinicalTrials.gov ID: NCT03311776) study [16]. The BIOPAC study is a prospective multicenter open cohort study with ongoing enrollment. The patients in this study were enrolled at five hospitals: Copenhagen University Hospital at Herlev (50\%, houses the biobank), Hillerød Hospital (6\%), Zealand University Hospital at Næstved (9\%), Odense University Hospital (10\%) and Copenhagen University Hospital at Rigshospitalet (25\%). Because Rigshospitalet is the most highly specialized hospital in Denmark and the largest one in volume with expertise in pancreatic cancer surgery, $71 \%$ of patients enrolled at Rigshospitalet were operated on. As operated patients usually have a smaller tumor burden and a better prognosis than patients who are not operated on, this likely explains why patients enrolled at Rigshospitalet had somewhat lower plasma levels of inflammatory biomarkers. However, no other patient characteristics differed between the hospitals. The enrollment period was from 3 July 2008 to 24 August 2017. The patients were followed until 4 October 2020, or death, whichever came first, for at least three years. Information on death was obtained from the national Danish Civil Registration System. The patients were operated on and/or treated with different types of chemotherapy according to national guidelines (www.gicancer.dk, accessed on 2 August 2021).

\subsection{Biochemical Analyses}

The blood for biochemical analyses was drawn around the time of diagnosis. For patients with stage II-IV PDAC, blood was drawn just prior to the commencement of 
chemotherapy (median (25th-75th percentile): 0 (0-0) days). For patients who were operated on $(\approx 30 \%)$, it was drawn prior to the operation (0 (0-34) days). The biochemical analyses of CRP $(n=975)$, CA 19-9 $(n=977)$, IL-6 $(n=993)$ and YKL-40 $(n=993)$ were performed on the same serum sample per patient. The levels of CRP were measured by turbidimetry (Dako, Glostrup, Denmark) using a standard laboratory assay, subjected to internal (daily) and external (monthly) quality control programs. CA 19-9 was analyzed in serum samples using a solid phase, two-site sequential chemiluminescent immunometric assay, the Immulite 2000 GI-MA (catalogue Number L2KG12, Siemens, Ballerup, Denmark). Due to some extremely high CA 19-9 measurements (up to 1,665,063 kU/L), we set an upper limit for CA 19-9 quantification levels to 100,000 kU/L. IL-6 and YKL-40 were determined in serum samples in duplicates by commercial two-site, sandwich-type enzyme-linked immunosorbent assays (IL-6: Catalogue number HS600, R\&D Systems, Abingdon, Oxon, UK; YKL-40: Quidel Corporation, San Diego, CA, USA). The detection limit was $0.01 \mathrm{ng} / \mathrm{L}$ for IL-6 and $20 \mu \mathrm{g} / \mathrm{L}$ for YKL-40.

\subsection{Covariates}

Information on baseline covariates was collected from patient records at the time of enrollment, i.e., blood sampling. We used participant-reported information on smoking habits and alcohol consumption. High alcohol consumption was defined as alcohol intake above 7 and 14 drinks per week for women and men, respectively ( 1 drink $\approx 12 \mathrm{~g}$ of alcohol). Body mass index was calculated by measured weight in kilograms (at enrollment) divided by measured height in meters squared. We calculated Charlson's age adjusted comorbidity index as the Charlson's comorbidity index after adding one point for each 10-year increase from 40 years of age [17]. Other covariates were performance status (PS), diabetes, operation, the presence of metastasis, tumor size and PDAC stage.

\subsection{Statistical Analysis}

We stratified the biomarkers into low, intermediate and high categories (percentage of the patients): CRP into $<10 \mathrm{mg} / \mathrm{L}(51 \%), 10-100 \mathrm{mg} / \mathrm{L}(40 \%)$ and $\geq 100 \mathrm{mg} / \mathrm{L}(9 \%)$; CA 19-9 into $<37 \mathrm{kU} / \mathrm{L}(19 \%), 37-10,000 \mathrm{kU} / \mathrm{L}(67 \%)$ and $\geq 10,000 \mathrm{kU} / \mathrm{L}(14 \%) ;$ IL-6 into $<5 \mathrm{ng} / \mathrm{L}(47 \%), 5-25 \mathrm{ng} / \mathrm{L}(43 \%)$ and $\geq 25 \mathrm{ng} / \mathrm{L}(10 \%) ;$ and YKL-40 into $<200 \mu \mathrm{g} / \mathrm{L}(68 \%)$, $200-799 \mu \mathrm{g} / \mathrm{L}(27 \%)$ and $\geq 800 \mu \mathrm{g} / \mathrm{L}(5 \%)$.

For CRP, CA 19-9 and IL-6, we chose commonly used routine cut-off levels, levels previously reported in the literature $[7,18]$ and/or rounded even levels where approximately $10 \%$ of patients were in the high category. For YKL- 40 , the low and high cut-offs at $<200 \mu \mathrm{g} / \mathrm{L}$ and $\geq 800 \mu \mathrm{g} / \mathrm{L}$ corresponded with previously used levels [19].

Interaction (between biomarkers and PS, operation and PDAC stage) was examined by the likelihood ratio test after linear regression between the models with and without an interaction term.

For each patient, we calculated a biomarker score $_{4}$ as the sum of each of the four biomarkers, coded 0, 1 and 2 for low, intermediate and high biomarker categories, respectively. Thus, patients received a biomarker score ${ }_{4}$ between 0 (if all four biomarkers were low) and 8 (if all four biomarkers were high). Additionally, we excluded plasma YKL-40 from the biomarker score 4 (as plasma YKL-40 was not associated with survival when adjusted for the three other biomarkers) and generated a new biomarker score ${ }_{3}$ of three biomarkers: CRP, CA 19-9 and IL-6 (score between 0 and 6).

Moreover, we used logarithmic base two transformations of CRP, CA 19-9, IL-6 and YKL-40 levels to estimate survival for a two-fold increase, i.e., doubling.

Because patients with PDAC have a very low five-year survival, we investigated cumulative survival for up to five years and the risk of one-year mortality. We used the Kaplan-Meier survival function to plot five-year cumulative survival curves, overall and stratified by PS (PS $=0$ versus PS $\geq 1$ ), operation (yes/no) and PDAC stage (stages I-II versus III-IV). The differences across biomarker categories and other categories of covariates were examined using log-rank trend tests. We used Cox regression analysis 
(with time since blood sampling as the time scale) to calculate hazard ratios (HRs) and 95\% confidence intervals (CIs) for one-year mortality after PDAC.

Model 1 was adjusted for age and sex and Models 2 and 3 additionally for PS (PS $\leq 1$ or PS $\geq 2$ ), operation (yes/no), cancer stage (I-II, III or IV) and the three biomarkers other than the one examined. Model 2 included only patients with complete information on these covariates, while Model 3 included all patients after multiple imputation of missing covariates. We had information on age, sex, operation and plasma IL-6 and YKL-40 measurements on all participants. We imputed missing values (Table 1$)$ of CRP $(n=18)$ by negative binomial regression; values of CA 19-9 $(n=16)$ by linear regression; and values of cancer stage $(n=11)$, PS $(n=96)$ and biomarker category $(n=18$ for CRP and $n=16$ for CA 19-9) by ordered logistic regression models using substantive model compatible fully conditional specification [20].

Table 1. Characteristics of patients with pancreatic ductal adenocarcinoma according to survival.

\begin{tabular}{|c|c|c|c|c|}
\hline Patient Characteristics & All & $\leq 1$ Year Survival & $>1$ Year Survival & Missing, $\%$ \\
\hline Number of participants, N (\%) & $993(100 \%)$ & $603(61 \%)$ & $390(39 \%)$ & NA \\
\hline Age, years & $67(61-72)$ & $67(61-73)$ & $67(61-72)$ & 0 \\
\hline Women, \% & 45 & 44 & 48 & 0 \\
\hline Eversmoker, \% & 61 & 61 & 62 & 10 \\
\hline Body mass index, $\mathrm{kg} / \mathrm{m}^{2}$ & $23.1(20.7-25.8)$ & $23.2(20.7-25.9)$ & $22.8(20.7-25.7)$ & 5.8 \\
\hline High alcohol consumption, $\%$ & 23 & 22 & 23 & 10 \\
\hline Performance status & $1(0-1)$ & $1(0-1)$ & $0(0-1)$ & 9.7 \\
\hline Charlson comorbidity index & $3(2-4)$ & $3(2-4)$ & $3(2-4)$ & 3.7 \\
\hline Diabetes, $\%$ & 26 & 26 & 25 & 1.8 \\
\hline Operated, \% & 30 & 16 & 52 & 0 \\
\hline Metastasing cancer, $\%$ & 50 & 65 & 27 & 0.5 \\
\hline Tumour size, mm & $3.5(2.5-4.8)$ & $4.0(3.0-5.0)$ & $3.0(2.3-4.0)$ & 13 \\
\hline Stage I-II, \% & 21 & 9.6 & 39 & 1.5 \\
\hline Stage III, \% & 27 & 23 & 34 & 0.8 \\
\hline Stage IV, \% & 51 & 66 & 27 & 0 \\
\hline C-reactive protein, $\mathrm{mg} / \mathrm{L}$ & $9(3-37)$ & $17(5-52)$ & $4(3-11)$ & 1.8 \\
\hline CA $19-9, \mathrm{kU} / \mathrm{L}$ & $445(59-3126)$ & $997(148-6290)$ & $105(29-796)$ & 1.6 \\
\hline Interleukin-6, $\mu \mathrm{g} / \mathrm{L}$ & $5.4(2.7-12)$ & $7.2(3.9-16)$ & $3.3(2.0-6.1)$ & 0 \\
\hline YKL-40, $\mu \mathrm{g} / \mathrm{L}$ & $138(81-227)$ & $156(92-246)$ & $113(67-205)$ & 0 \\
\hline
\end{tabular}

Values collected at inclusion from 3 July 2008 to 24 August 2017, are expressed as numbers of participants, frequencies or medians (interquartile ranges). Numbers of participants across listed variables vary slightly because we did not have information on all participants for all listed variables. NA = not applicable.

The predictive values of plasma biomarkers for one-year mortality were examined by area under the receiver operating characteristic curves (AUC).

\subsection{Additional Analyses}

We investigated pairwise combinations of CRP, CA 19-9, IL-6 and YKL-40. Furthermore, in order to investigate the recently proposed prognostic score [15], we grouped CRP and CA 19-9 levels into (1) CRP $<3 \mathrm{mg} / \mathrm{L}$ and CA $19-9 \leq 37 \mathrm{kU} / \mathrm{L}$, (2) CRP $\geq 3 \mathrm{mg} / \mathrm{L}$ or CA 19-9 $>37 \mathrm{kU} / \mathrm{L}$ and (3) CRP $\geq 3 \mathrm{mg} / \mathrm{L}$ and CA 19-9 > 37 kU/L. Finally, additional analyses regarding measured as well as genetically predicted plasma YKL-40 (CHI3L1 rs4950928 genotype) in relation to CRP, CA-19-9 and IL-6 are described in Supplementary Methods [19,21-32].

\section{Results}

Baseline characteristics are shown in Table 1 . Thirty percent of the patients were operated on, as their tumor was considered resectable. During the study period, 947 patients ( $95 \%)$ died. The median follow-up time was 264 days (25-75th percentile: $129-557$ days). The five-year survival was only $6.3 \%$ (63 out of 993 participants). 


\subsection{Plasma CRP, CA 19-9, IL-6, YKL-40 and Survival}

For the increasing categories of all four biomarkers, overall survival after PDAC decreased (Figures 1 and S1-S4).
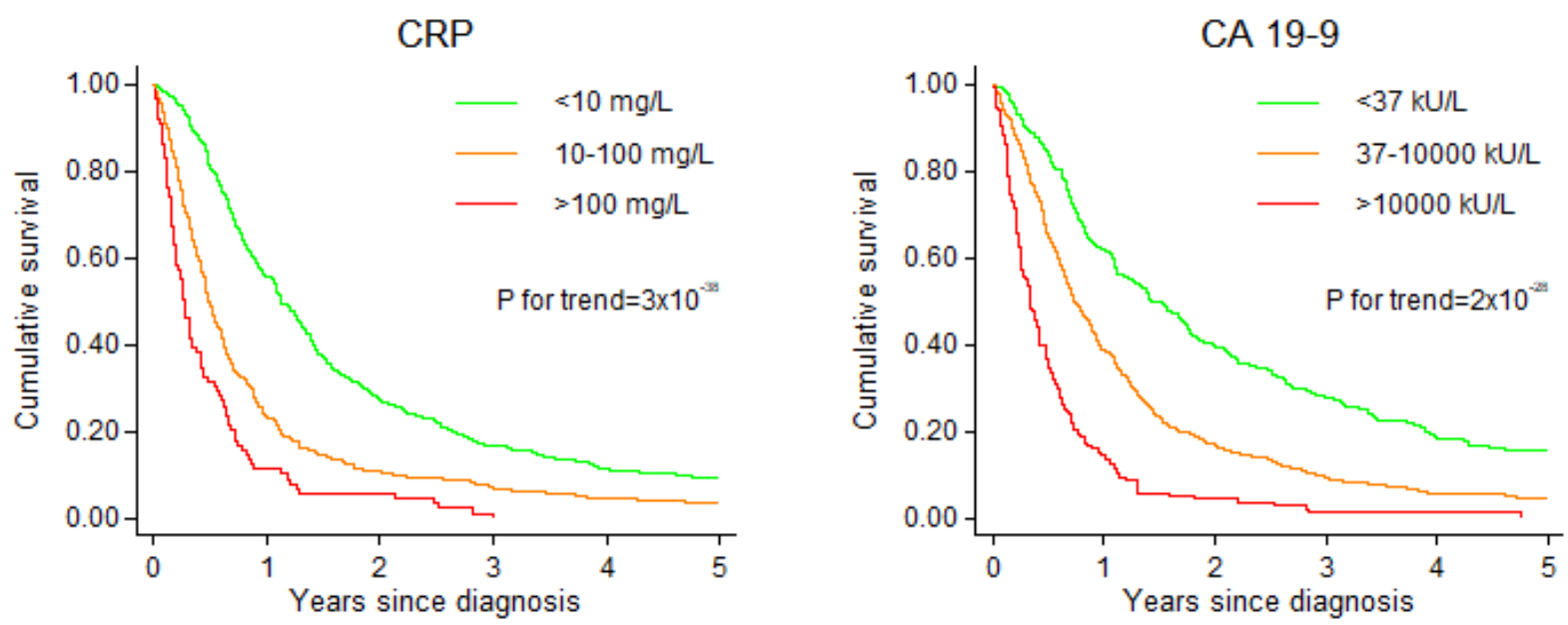

Number at risk by
biomarker levels:

$\begin{array}{rccccc}<10 \mathrm{mg} / \mathrm{L} 496 & 276 & 136 & 82 & 50 & 30 \\ 10-100 \mathrm{mg} / \mathrm{L} 390 & 92 & 42 & 28 & 15 & 10 \\ >100 \mathrm{mg} / \mathrm{L} 89 & 10 & 5 & 1 & 0 & 0\end{array}$

Number at risk by

$\begin{array}{rccccc}<37 \mathrm{kU} / \mathrm{L} 186 & 115 & 73 & 52 & 29 & 18 \\ 37-10000 \mathrm{kU} / \mathrm{L} 653 & 252 & 110 & 62 & 36 & 22 \\ >10000 \mathrm{kU} / \mathrm{L} 138 & 20 & 6 & 2 & 1 & 0\end{array}$

\section{IL-6}
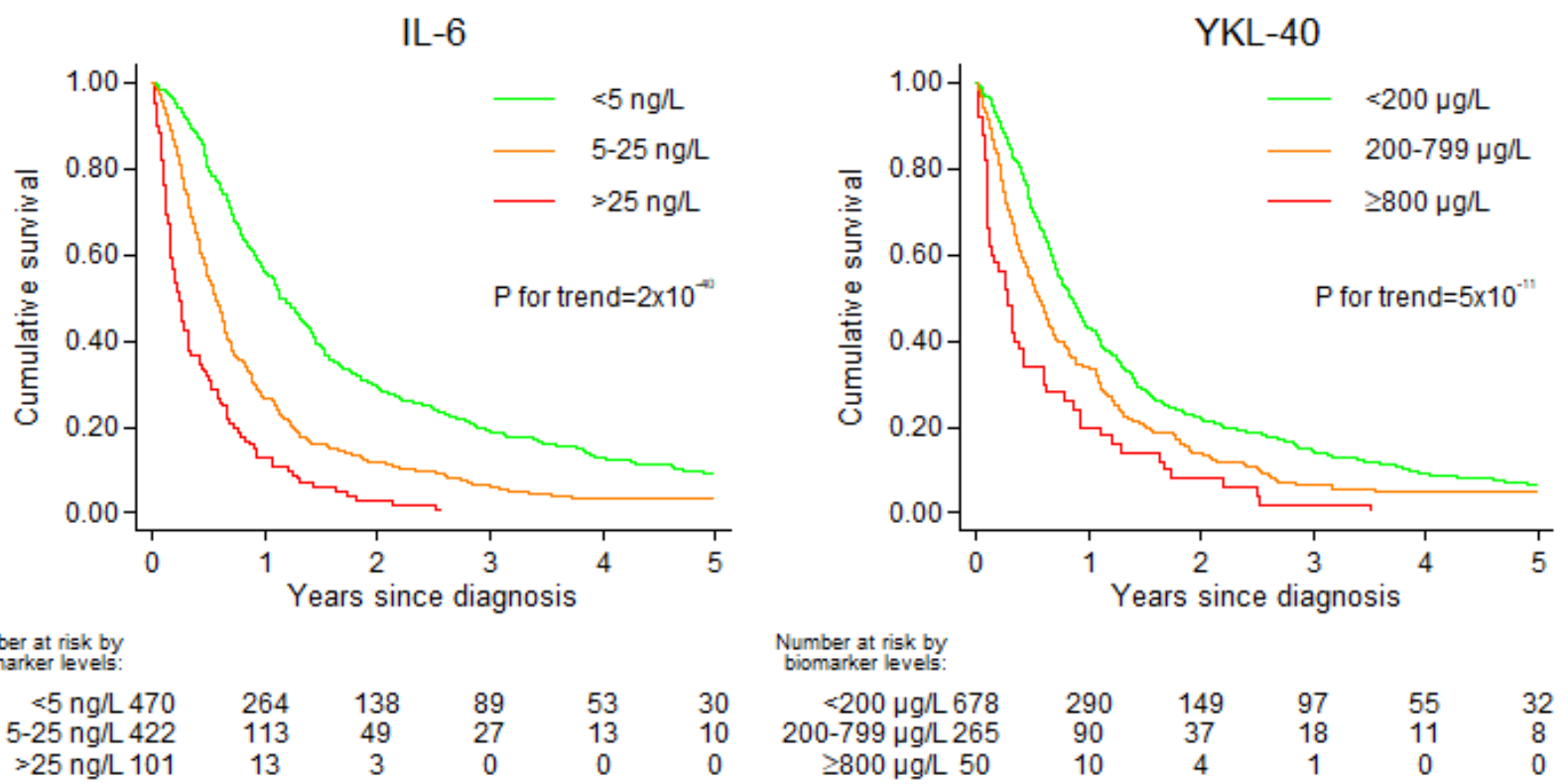

Figure 1. Kaplan-Meier survival curves for plasma CRP, CA 19-9, IL-6 and YKL-40 categories. $p$-value for trend is from Wald test of trend across groups.

Poorer PS (PS $\geq 1$ versus PS $=0$ ), not being operated (versus being operated) on and a higher PDAC stage (stage III-IV versus stage I-II) were associated with decreased survival (Figures S1-S4). We observed no robust interactions between the four biomarkers and PS, operation and PDAC stage. HRs (Model 3) for one-year mortality were 2.20 (95\% confidence interval: 1.62-2.99) for high versus low CRP, 2.13 (1.56-2.92) for high versus low CA 19-9, 2.08 (1.51-2.89) for high versus low IL-6 and 1.07 (0.74-1.54) for high versus low plasma YKL-40 (Figure 2). 


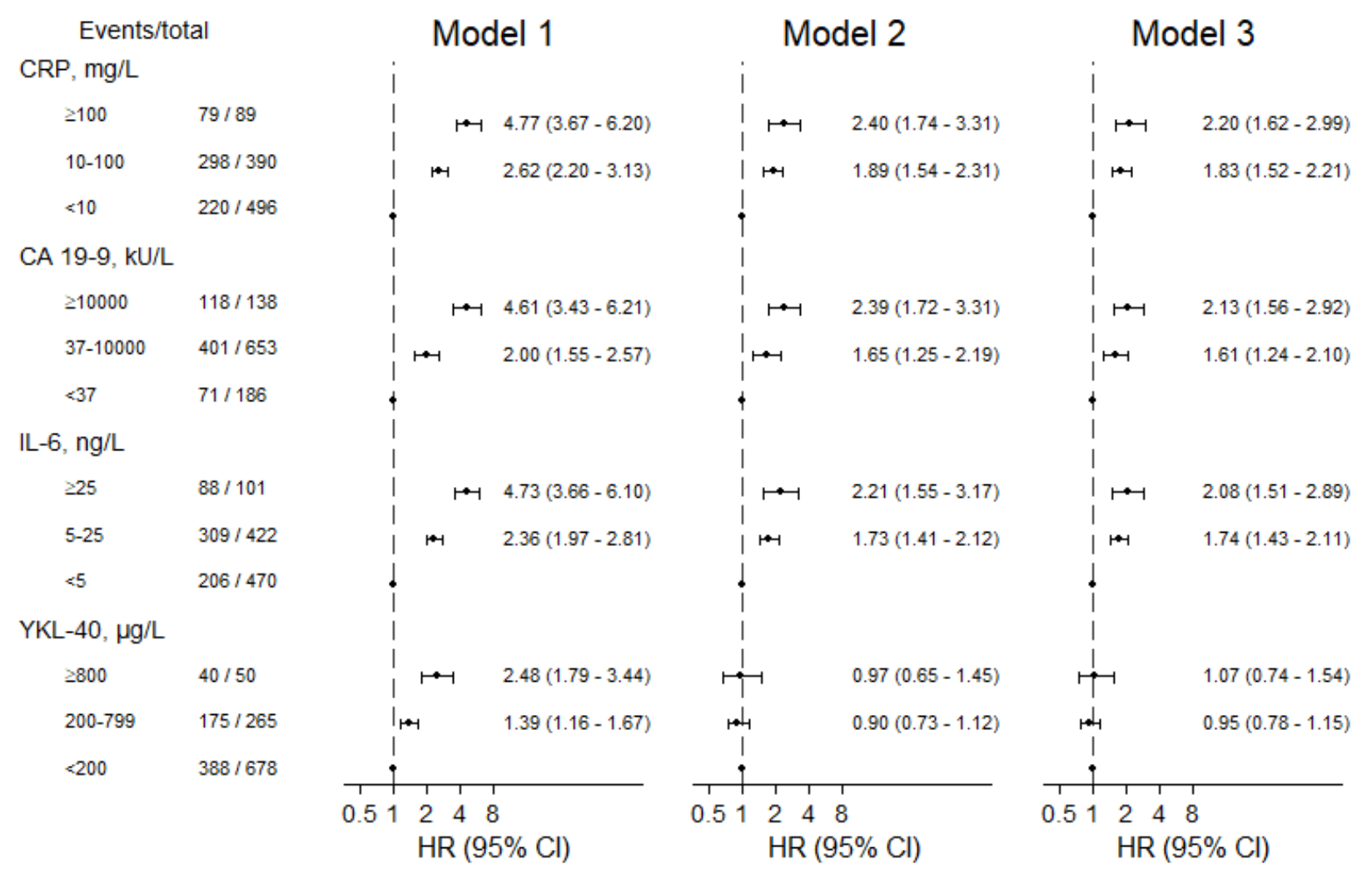

Figure 2. Risk of one-year mortality after diagnosis of pancreatic ductal adenocarcinoma according to biomarker categories. Model 1 was adjusted for age and sex and included all participants. Models 2 and 3 were additionally adjusted for performance status (0-1 and 2-4), operation (yes/no), cancer stage (I-II and III-IV) and all the biomarkers (except if considered exposure). Model 2 included patients with complete information and Model 3 all patients due to multiple imputation.

The HRs (Model 3) for one-year mortality per doubling in plasma biomarker levels were 1.18 (1.13-1.24) for CRP, 1.07 (1.05-1.10) for CA 19-9, 1.23 (1.15-1.31) for IL-6 and 1.06 (0.98-1.14) for YKL-40 (Figure S5).

In accordance with the observation that elevated plasma YKL-40 was not independently associated with poor survival after adjustment for the three other biomarkers, genetically elevated YKL-40 (chitinase-3-like-1 (CHI3L1) rs4950928 genotype) was not associated with poor survival either (Figure S6).

CRP, CA 19-9 and IL-6 were comparable with regard to the prediction of one-year mortality using the area under the receiver operating characteristic curves (AUROC), while the corresponding values for plasma YKL-40 were consistently smaller than those for any other biomarker (Figure 3).

CRP (and IL-6) had the best one-year survival predictive ability for patients who were not operated on and had stage III-IV PDAC, while CA 19-9 had the best one-year survival predictive ability for patients who were operated on and had stage I-II PDAC (Figure 3). When we restricted the analyses to participants who had measurements of all four biomarkers, the results were similar (Figure S7).

\subsection{Plasma CRP, CA 19-9, IL-6 and YKL-40: Biomarker Score}

Increasing categories of biomarker score were associated with increased mortality (Figures 4 and S8).

Compared to the biomarker score $4=0$, the HRs (Model 3) for one-year mortality were $1.44(0.90-2.28)$ for score $4=1,1.67(1.05-2.66)$ for score $_{4}=2,2.81(1.77-4.49)$ for score $_{4}=3,3.48(2.18-5.56)$ for score $4=4,4.62(2.80-7.62)$ for score $4=5$ and $5.49(3.30-9.13)$ for score $4=6-8 ; p$-trend $=7 \times 10^{-28}$ (Figure S8), corresponding to an HR of 1.34 (1.27-1.41) for each unit increase in biomarker score 4 . 

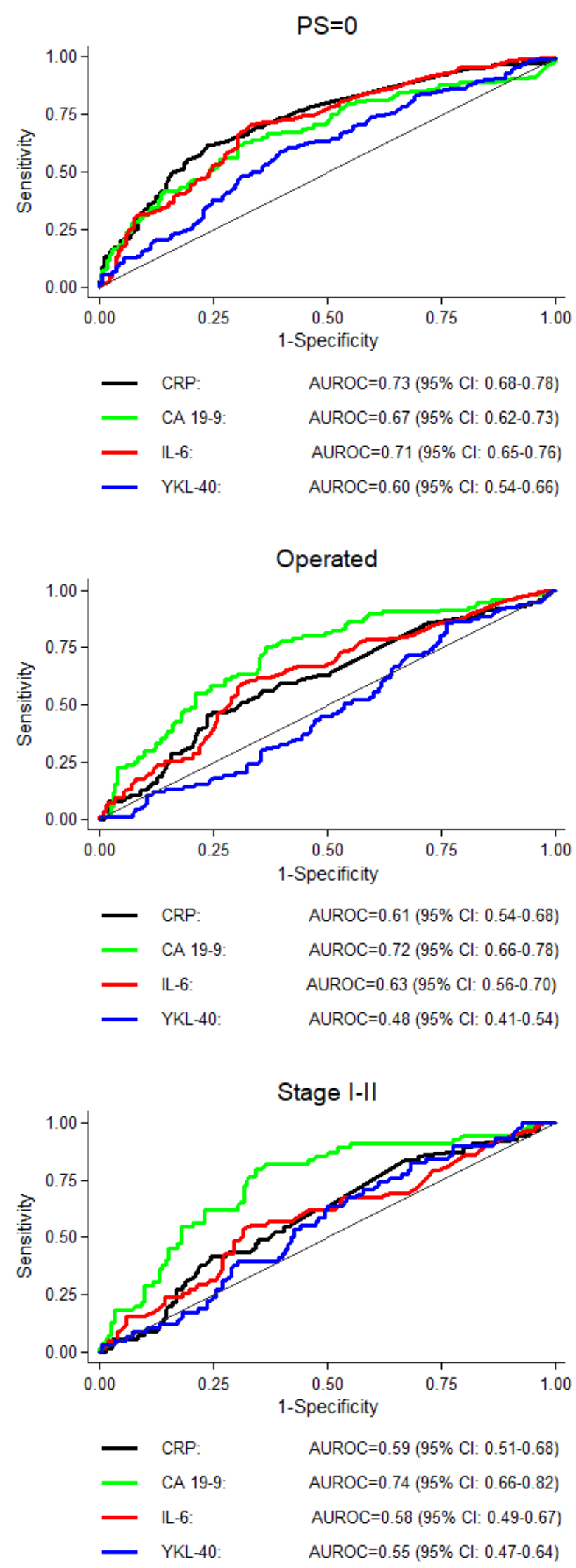
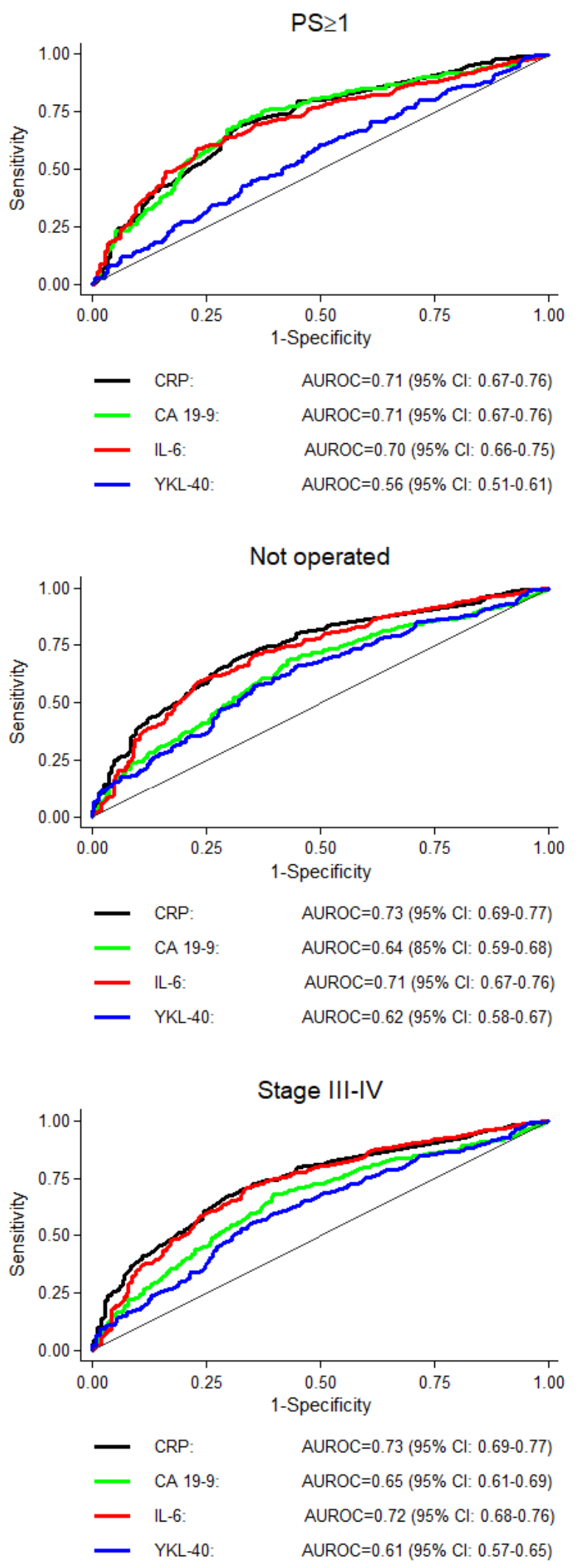

Figure 3. Area under the receiver operating characteristic (AUROC) curves for one-year mortality for CRP, CA 19-9, IL-6 and YKL-40, stratified by performance status (PS), operation (yes/no) and pancreatic ductal adenocarcinoma stage. 


\section{Biomarker score $:$ CRP, CA 19-9 and IL-6}

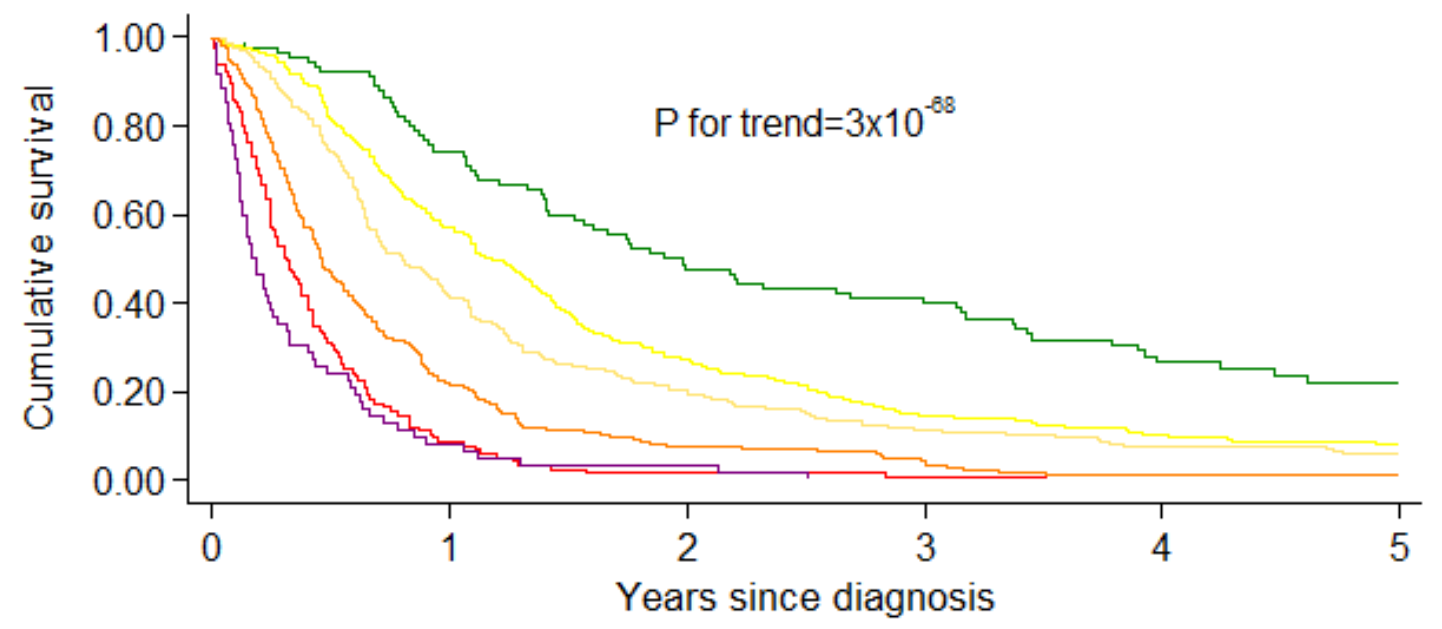

Number at risk by biomarker score $_{3}$.

\begin{tabular}{lccccccc}
$\square$ & 0 & 90 & 67 & 43 & 36 & 20 & 11 \\
- & 1 & 295 & 168 & 81 & 43 & 28 & 18 \\
$\square$ & 2 & 192 & 80 & 38 & 22 & 14 & 9 \\
$\square$ & 3 & 205 & 45 & 16 & 9 & 3 & 2 \\
$\square$ & 4 & 115 & 10 & 2 & 1 & 0 & 0 \\
\hline & $5-6$ & 62 & 5 & 2 & 0 & 0 & 0
\end{tabular}

Figure 4. Kaplan-Meier survival curve for plasma CRP, CA 19-9 and IL-6 categories combined into a biomarker score 3 . Biomarker score $_{3}$ was calculated as the sum of CRP, CA 19-9 and IL-6 categories coded as 0, 1 and 2 for low, intermediate and high plasma levels, respectively. $p$-value for trend is from Wald test of trend across groups.

When we excluded YKL-40 from the biomarker score 4 , i.e., restricted the score 3 to CRP, CA 19-9 and IL-6, the estimates were augmented. Compared to the biomarker score $3=0$, the HRs for one-year mortality were $1.56(0.99-2.44)$ for score $_{3}=1,2.22(1.41-3.49)$ for score $_{3}=2,3.44(2.20-5.38)$ for score $3=3,5.13(3.21-8.17)$ for score $3=4$ and $6.32(3.84-10.41)$ for score $_{3}=5-6 ; p$-value for trend $=3 \times 10^{-31}$ (Figure 5), corresponding to an HR of 1.46 (1.37-1.55) for each unit increase in biomarker score 3 .

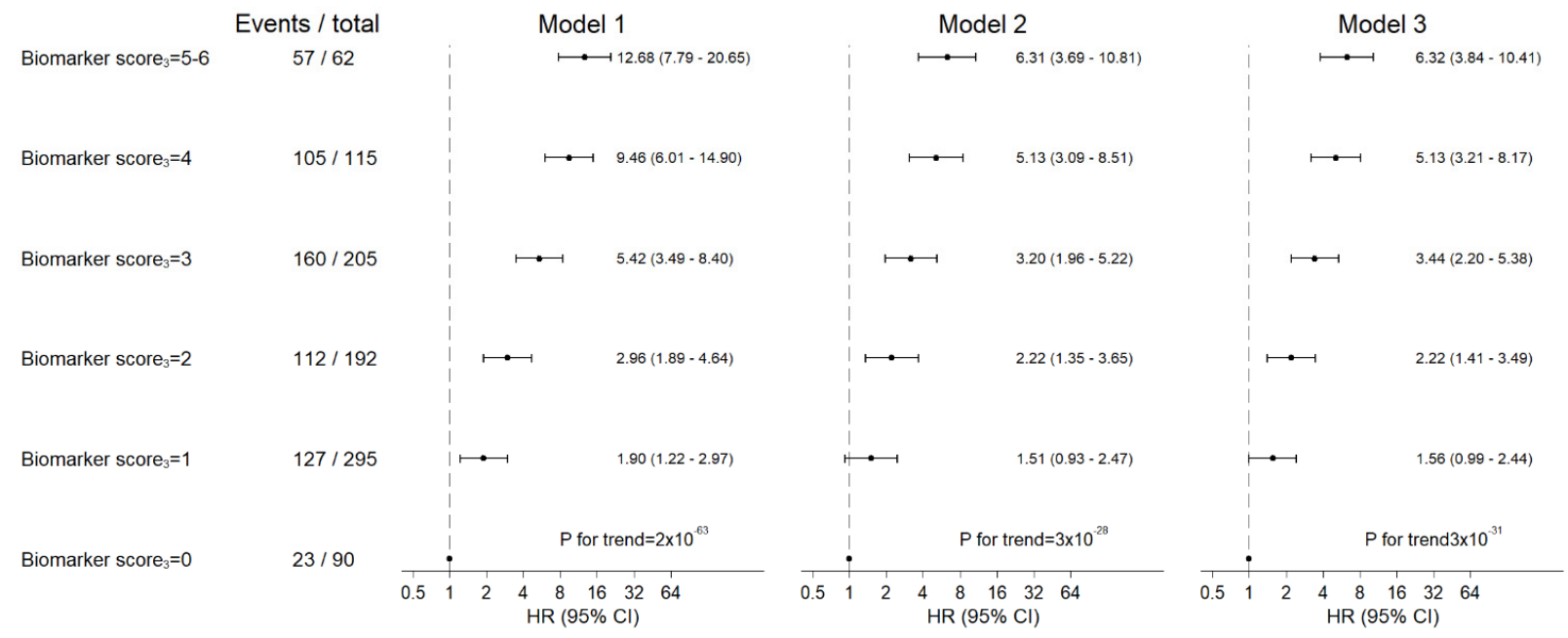

Figure 5. Risk of one-year mortality after pancreatic ductal adenocarcinoma according to biomarker score 3 . Biomarker score $_{3}$ was calculated as the sum of CRP, CA 19-9 and IL-6 categories coded as 0, 1 and 2 for low, intermediate and high plasma levels, respectively. $p$-values for trend are from Wald tests across groups treating biomarker levels as continuous variables in the Cox regression model. 
When we stratified the analyses by operation status, the risk estimates for one-year mortality were even higher nominally (but with very broad 95\% CIs) for the patients who were operated on compared to the patients who were not operated on (Figure S9). However, we found no evidence of interactions between biomarker score s $_{3}$ and PS, operation and PDAC stage (all $p$-values for interaction $\geq 0.09$ ).

Importantly, the inflammatory biomarker score 3 performed better than any of the four single biomarkers when examined in similarly sized or other categories.

\subsection{Additional Analyses}

The HRs (Model 3) for one-year mortality were similar for CRP in combination with either CA 19-9 or IL-6 (4.64 (2.60-8.30) and 4.69 (3.29-6.68) for score $=4$ versus score $=0$, respectively, Table S3). The corresponding estimates were somewhat lower for pairwise biomarker combinations with YKL-40 and somewhat higher for a combination of CA 19-9 and IL-6 but with largely overlapping confidence intervals, and, thus, they were not robustly different from each other (Table S3). Estimates were higher but imprecise for patients who were operated on compared to those who were not operated on (Table S3).

Compared to CRP $<3 \mathrm{mg} / \mathrm{L}$ and CA 19-9 $\leq 37 \mathrm{kU} / \mathrm{L}$, the HRs (Model 3) for one-year mortality were 1.51 (0.75-3.00) for CRP $\geq 3 \mathrm{mg} / \mathrm{L}$ or CA 19-9 > 37 kU/L and 2.62 (1.34-5.13) for CRP $\geq 3 \mathrm{mg} / \mathrm{L}$ and CA 19-9 > $37 \mathrm{kU} / \mathrm{L}$ (Table S4). Estimates were higher but imprecise for patients who were operated on compared to those who were not operated on (Table S4).

The results regarding plasma YKL-40 and CHI3L1 rs4950928 in relation to CRP, CA-199 and IL-6 are shown in Supplementary Results (Tables S1 and S2 and Figures S10 and S11).

\section{Discussion}

By studying 993 patients with PDAC from the BIOPAC study, we found that elevated plasma CRP, CA 19-9 and IL-6, but not YKL-40, were independently associated with poor survival. A combination score of elevated CRP, CA 19-9 and IL-6 identified patients with PDAC who had more than six-fold higher one-year mortality. Importantly, the biomarker score performed substantially better than each biomarker individually and pairwise. The ability of elevated CRP, CA 19-9 and IL-6 to predict one-year mortality, in terms of ROC statistics, was comparable to the ability of low-density lipoprotein cholesterol and apolipoprotein B to predict myocardial infarction [33].

Compared with previous studies based on the BIOPAC $[12,13]$, the present study included twice as many patients and had a longer follow-up time. Because $95 \%$ of the participants died during follow-up, we assessed one-year survival, which we had complete information on. Furthermore, we stratified cumulative survival and AUROC analyses by PS, operation and PDAC stage. Finally, while the previous studies dichotomized biomarkers, we grouped biomarker levels into three categories each.

Our results are largely in agreement with the previous studies because (1) YKL-40 was not robustly associated with overall survival; (2) CA 19-9 was superior to IL-6 as a prognostic marker for patients who were operated on, while the opposite was the case for patients who were not operated on; and (3) a combination of biomarkers was a better prognostic marker than each biomarker individually [12,13]. Importantly, a combination of CRP, IL-6 and CA 19-9, and thus without YKL-40, had the best prognostic performance with regard to one-year mortality, particularly for the patients who were operated on.

In the present study, CRP and IL-6 performed best as prognostic biomarkers for patients who were not operated on and had high stage PDAC, while CA 19-9 performed best as a prognostic biomarker for patients who were operated on and had low stage PDAC. A possible explanation could be that CRP and IL-6 compared with CA 19-9 may be more sensitive biomarkers of other inflammation than PDAC, e.g., infections. However, this is only speculative. Nonetheless, our findings suggest that for the $70 \%$ of patients with PDAC who are not operated on, there is no reason to favour CA 19-9 above CRP for prognostic purposes. This is in agreement with previous studies that showed similar performance of the two biomarkers [7,11,18,34,35]. Measuring CRP is inexpensive and 
easily available and could be particularly useful for the $5-10 \%$ of patients who do not produce plasma CA 19-9 [36]. However, plasma CA 19-9 has remained the most widely used biomarker for PDAC [14] and the only biomarker currently recommended for clinical use by the US National Comprehensive Cancer Network guidelines (https:/ / www.nccn. org/professionals/physician_gls/pdf/pancreatic.pdf, accessed on 2 August 2021).

Our results are largely in agreement with a recent Finnish study of 212 patients who were operated on for PDAC [15]. We found that elevated CRP ( $\geq 3 \mathrm{mg} / \mathrm{L})$ and/or CA 19-9 $(>37 \mathrm{kU} / \mathrm{L})$ was associated with a two- to five-fold increased risk of one-year mortality in patients who were operated on, but there was no robust association in those who were not.

The strengths of this study include its prospective design, the large number of patients with PDAC and the measurements of four biomarkers of each patient. It is also a strength that we had $100 \%$ follow-up; that is, we did not lose track of even a single patient.

The potential limitations of the present study include survival bias and selective nonresponse by individuals with poor PS and a high PDAC stage. However, the lack of patients with the most severe phenotype would likely bias the estimates toward the null, and the five-year survival of only $6.4 \%$ does not support this. Another limitation is that we were unable to perform a validation study of the biomarker score. Despite the relatively large size of this study, when we stratified patients according to operation status, the confidence intervals for biomarker score were very broad, particularly in the operated strata. According to the Danish national guidelines, a conventional CT scan is the most commonly used modality at the initial staging of PDAC. Thus, microscopic disease and small metastases under the detection level may have been overlooked. Since very few patients had an additional PET-CT performed, we were unable to investigate CA 19-9 levels according to CT modality (conventional versus PET-CT). Furthermore, not only PDAC but also many infections are associated with increased plasma levels of inflammatory biomarkers. However, since blood sampling was performed just prior to the commencement of chemotherapy or operation, both of which are contra-indicated in patients with overt infections, it is unlikely that infections affected plasma biomarker levels to a large degree. Finally, as we only included patients from the (predominantly white) Danish population, our findings do not necessarily apply to other ethnicities. Yet, to our knowledge, there are no data suggesting that our findings could not also be directly applicable to other ethnicities.

\section{Conclusions}

In this large prospective study of patients with PDAC, we found that CRP outperformed CA 19-9 for prognostic purposes in the 70\% of patients with PDAC who were not operated on. A combination of CRP, CA 19-9 and IL-6 into a single biomarker score 3 was the best prognostic marker and may be clinically valuable in identifying patients with PDAC who have the poorest prognosis.

Supplementary Materials: The following are available online at https:/ / www.mdpi.com/article/ 10.3390/cancers13184599/s1, Supplementary Introduction, Supplementary Methods, Supplementary References, Table S1: Characteristics of patients with pancreatic ductal adenocarcinoma according to plasma YKL-40 categories, Table S2: Characteristics of patients with pancreatic ductal adenocarcinoma according to CHI3L1 rs4950928, Table S3: Risk of one-year mortality after pancreatic ductal adenocarcinoma according to pairwise combinations of low, intermediate and high CRP, CA19-9, IL-6 and YKL-40 levels stratified by operation status, Table S4: Risk of one-year mortality after pancreatic ductal adenocarcinoma according to a combination of low and high CRP and CA19-9 levels stratified by operation status, Figure S1: Kaplan-Meier survivor function stratified by performance status (PS), operation and pancreatic ductal adenocarcinoma stage for plasma CRP categories, Figure S2: Kaplan-Meier survivor function stratified by performance status (PS), operation and pancreatic ductal adenocarcinoma stage for plasma CA-19-9 categories, Figure S3: Kaplan-Meier survivor function stratified by performance status (PS), operation and pancreatic ductal adenocarcinoma stage for plasma IL-6 categories, Figure S4: Kaplan-Meier survivor function stratified by performance status (PS), operation and pancreatic ductal adenocarcinoma stage for plasma YKL-40 
categories, Figure S5: Risk of one-year mortality after diagnosis of pancreatic ductal adenocarcinoma per doubling in biomarker levels, Figure S6: Kaplan-Meier survival curves for CHI3L1 rs4950928 genotype stratified by performance status (PS), operation and pancreatic ductal adenocarcinoma stage, Figure S7: Area under receiver operating curves (AUROC) for one-year mortality for CRP, CA 19-9, IL-6 and YKL-40 stratified by performance status (PS), operation and pancreatic ductal adenocarcinoma (PDAC) stage, Figure S8: Risk of one-year mortality after diagnosis of pancreatic ductal adenocarcinoma according to biomarker score (the sum of CRP, CA 19-9, IL-6 and YKL-40 coded as 0, 1 and 2 for low, intermediate and high plasma levels, respectively, Figure S9: Risk of one-year mortality after diagnosis of pancreatic ductal adenocarcinoma according to biomarker score (the sum of CRP, CA 19-9, and IL-6 coded as 0, 1 and 2 for low, intermediate and high plasma levels, respectively, and operation, Figure S10: Median plasma YKL-40 by CHI3L1 rs4950928 genotype and population, Figure S11: Median levels of C-reactive protein, interleukin-6 and CA 19-9 across the plasma YKL-40 categories.

Author Contributions: Conceptualization, A.D.K., B.G.N., S.E.B. and J.S.J.; data curation, A.Z.J.; formal analysis, A.D.K.; investigation, I.M.C. and J.S.J.; methodology, A.D.K.; project administration, J.S.J.; resources, I.M.C. and J.S.J.; supervision, B.G.N., S.E.B. and J.S.J.; visualization, A.D.K.; writingoriginal draft, A.D.K.; writing-review and editing, I.M.C., A.Z.J., B.G.N., S.E.B. and J.S.J. All authors have read and agreed to the published version of the manuscript.

Funding: This research received no external funding.

Institutional Review Board Statement: The study was conducted according to the guidelines of the Declaration of Helsinki and approved by the Danish Ethics Committee (VEK, j.nr. KA-20060113) and the Danish Data Protection Agency (j.nr. 2012-58-0004; HGH-2015-027; I-Suite j.nr. 03960 and PACTIUS P-2020-834).

Informed Consent Statement: Informed consent was obtained from all subjects involved in the study.

Data Availability Statement: The data presented in this study are not publicly available due to the Danish legislature, e.g., for the privacy of individuals that participated in the study.

Acknowledgments: Many thanks to the biomedical laboratory scientists Charlotte Falk and Vibeke H. Holm, Ulla Kjærulff-Hansen, Teresa Rozenfeld and Mie B. Krüger for their assistance with the biobank and analysis of YKL-40 and IL-6. Medical doctors and nurses at Herlev and Gentofte Hospital, Hillerød Hospital, Næstved Hospital, Odense University Hospital, Rigshospitalet and Aalborg University Hospital are acknowledged for their contribution to inclusions of patients in the BIOPAC protocol.

Conflicts of Interest: The authors declare no conflict of interest.

\section{References}

1. Bray, F.; Ferlay, J.; Soerjomataram, I.; Siegel, R.L.; Torre, L.A.; Jemal, A. Global cancer statistics 2018: GLOBOCAN estimates of incidence and mortality worldwide for 36 cancers in 185 countries. CA Cancer J. Clin. 2018, 68, 394-424. [CrossRef] [PubMed]

2. Rahib, L.; Smith, B.D.; Aizenberg, R.; Rosenzweig, A.B.; Fleshman, J.M.; Matrisian, L.M. Projecting cancer incidence and deaths to 2030: The unexpected burden of thyroid, liver, and pancreas cancers in the United States. Cancer Res. 2014, 74, $2913-2921$. [CrossRef] [PubMed]

3. Gordon-Dseagu, V.L.; Devesa, S.S.; Goggins, M.; Stolzenberg-Solomon, R. Pancreatic cancer incidence trends: Evidence from the Surveillance, Epidemiology and End Results (SEER) population-based data. Int. J. Epidemiol. 2018, 47, 427-439. [CrossRef] [PubMed]

4. $\quad$ Kleeff, J.; Korc, M.; Apte, M.; La Vecchia, C.; Johnson, C.D.; Biankin, A.V.; Neale, R.E.; Tempero, M.; Tuveson, D.A.; Hruban, R.H.; et al. Pancreatic cancer. Nat. Rev. Dis. Primers 2016, 2, 16022. [CrossRef]

5. Crawford, H.C.; Pasca di Magliano, M.; Banerjee, S. Signaling Networks That Control Cellular Plasticity in Pancreatic Tumorigenesis, Progression, and Metastasis. Gastroenterology 2019, 156, 2073-2084. [CrossRef] [PubMed]

6. Babic, A.; Schnure, N.; Neupane, N.P.; Zaman, M.M.; Rifai, N.; Welch, M.W.; Brais, L.K.; Rubinson, D.A.; Morales-Oyarvide, V.; Yuan, C.; et al. Plasma inflammatory cytokines and survival of pancreatic cancer patients. Clin. Transl. Gastroenterol. $2018,9,145$. [CrossRef]

7. Haas, M.; Heinemann, V.; Kullmann, F.; Laubender, R.P.; Klose, C.; Bruns, C.J.; Holdenrieder, S.; Modest, D.P.; Schulz, C.; Boeck, S. Prognostic value of CA 19-9, CEA, CRP, LDH and bilirubin levels in locally advanced and metastatic pancreatic cancer: Results from a multicenter, pooled analysis of patients receiving palliative chemotherapy. J. Cancer Res. Clin. Oncol. 2013, 139, 681-689. [CrossRef] 
8. Mitsunaga, S.; Ikeda, M.; Shimizu, S.; Ohno, I.; Takahashi, H.; Okuyama, H.; Ueno, H.; Morizane, C.; Kondo, S.; Sakamoto, Y.; et al. C-Reactive Protein Level Is an Indicator of the Aggressiveness of Advanced Pancreatic Cancer. Pancreas 2016, 45, 110-116. [CrossRef]

9. Szkandera, J.; Stotz, M.; Absenger, G.; Stojakovic, T.; Samonigg, H.; Kornprat, P.; Schaberl-Moser, R.; Alzoughbi, W.; Lackner, C.; Ress, A.L.; et al. Validation of C-reactive protein levels as a prognostic indicator for survival in a large cohort of pancreatic cancer patients. Br. J. Cancer 2014, 110, 183-188. [CrossRef]

10. Vainer, N.; Dehlendorff, C.; Johansen, J.S. Systematic literature review of IL-6 as a biomarker or treatment target in patients with gastric, bile duct, pancreatic and colorectal cancer. Oncotarget 2018, 9, 29820-29841. [CrossRef]

11. Xue, P.; Zhu, L.; Wan, Z.; Huang, W.; Li, N.; Chen, D.; Hu, J.; Yang, H.; Wang, L. A prognostic index model to predict the clinical outcomes for advanced pancreatic cancer patients following palliative chemotherapy. J. Cancer Res. Clin. Oncol. 2015, 141, 1653-1660. [CrossRef]

12. Chen, I.M.; Johansen, A.Z.; Dehlendorff, C.; Jensen, B.V.; Bojesen, S.E.; Pfeiffer, P.; Bjerregaard, J.K.; Nielsen, S.E.; Andersen, F.; Hollander, N.H.; et al. Prognostic Value of Combined Detection of Serum IL6, YKL-40, and C-reactive Protein in Patients with Unresectable Pancreatic Cancer. Cancer Epidemiol. Biomark. Prev. 2020, 29, 176-184. [CrossRef] [PubMed]

13. Schultz, N.A.; Christensen, I.J.; Werner, J.; Giese, N.; Jensen, B.V.; Larsen, O.; Bjerregaard, J.K.; Pfeiffer, P.; Calatayud, D.; Nielsen, S.E.; et al. Diagnostic and Prognostic Impact of Circulating YKL-40, IL-6, and CA 19.9 in Patients with Pancreatic Cancer. PLoS ONE 2013, 8, e67059. [CrossRef]

14. Khomiak, A.; Brunner, M.; Kordes, M.; Lindblad, S.; Miksch, R.C.; Öhlund, D.; Regel, I. Recent Discoveries of Diagnostic, Prognostic and Predictive Biomarkers for Pancreatic Cancer. Cancers 2020, 12, 3234. [CrossRef]

15. Nurmi, A.M.; Mustonen, H.K.; Stenman, U.H.; Seppänen, H.E.; Haglund, C.H. Combining CRP and CA19-9 in a novel prognostic score in pancreatic ductal adenocarcinoma. Sci. Rep. 2021, 11, 781. [CrossRef]

16. Chen, I.; Vittrup Jensen, B.; Bojesen, S.E.; Johansen, A.Z.; Schultz, N.A.; Hansen, C.P.; Hasselby, J.P.; Holländer, N.H.; Nissen, M.H.B.; Bjerregaard, J.K.; et al. Identification of New Biomarkers in Patients with Pancreatic Cancer (BIOPAC): A Study Protocol of an Open Cohort Study. J. Cancer Sci. Ther. 2019, 11, 232-239.

17. Charlson, M.E.; Pompei, P.; Ales, K.L.; MacKenzie, C.R. A new method of classifying prognostic comorbidity in longitudinal studies: Development and validation. J. Chronic Dis. 1987, 40, 373-383. [CrossRef]

18. Ueno, H.; Okada, S.; Okusaka, T.; Ikeda, M. Prognostic factors in patients with metastatic pancreatic adenocarcinoma receiving systemic chemotherapy. Oncology 2000, 59, 296-301. [CrossRef] [PubMed]

19. Kjaergaard, A.D.; Bojesen, S.E.; Nordestgaard, B.G.; Johansen, J.S. YKL-40 and alcoholic liver and pancreas damage and disease in 86,258 individuals from the general population: Cohort and mendelian randomization studies. Clin. Chem. 2014, 60, 1429-1440. [CrossRef]

20. Bartlett, J.W.; Seaman, S.R.; White, I.R.; Carpenter, J.R.; Alzheimer's Disease Neuroimaging, I. Multiple imputation of covariates by fully conditional specification: Accommodating the substantive model. Stat. Methods Med. Res. 2015, 24, 462-487. [CrossRef]

21. Chen, Y.; Zhang, S.; Wang, Q.; Zhang, X. Tumor-recruited M2 macrophages promote gastric and breast cancer metastasis via M2 macrophage-secreted CHI3L1 protein. J. Hematol. Oncol. 2017, 10, 36. [CrossRef]

22. Cohen, N.; Shani, O.; Raz, Y.; Sharon, Y.; Hoffman, D.; Abramovitz, L.; Erez, N. Fibroblasts drive an immunosuppressive and growth-promoting microenvironment in breast cancer via secretion of Chitinase 3-like 1. Oncogene 2017, 36, 4457-4468. [CrossRef] [PubMed]

23. Dimitrakopoulos, C.; Vrugt, B.; Flury, R.; Schraml, P.; Knippschild, U.; Wild, P.; Hoerstrup, S.; Henne-Bruns, D.; Wuerl, P.; Graf, R.; et al. Identification and Validation of a Biomarker Signature in Patients With Resectable Pancreatic Cancer via Genome-Wide Screening for Functional Genetic Variants. JAMA Surg. 2019, 154, e190484. [CrossRef] [PubMed]

24. Geng, B.; Pan, J.; Zhao, T.; Ji, J.; Zhang, C.; Che, Y.; Yang, J.; Shi, H.; Li, J.; Zhou, H.; et al. Chitinase 3-like 1-CD44 interaction promotes metastasis and epithelial-to-mesenchymal transition through beta-catenin/Erk/Akt signaling in gastric cancer. J. Exp. Clin. Cancer Res. 2018, 37, 208. [CrossRef] [PubMed]

25. Huang, W.S.; Lin, H.Y.; Yeh, C.B.; Chen, L.Y.; Chou, Y.E.; Yang, S.F.; Liu, Y.F. Correlation of Chitinase 3-Like 1 Single Nucleotide Polymorphisms with Hepatocellular Carcinoma in Taiwan. Int. J. Med. Sci. 2017, 14, 136-142. [CrossRef]

26. Kim, D.H.; Park, H.J.; Lim, S.; Koo, J.H.; Lee, H.G.; Choi, J.O.; Oh, J.H.; Ha, S.J.; Kang, M.J.; Lee, C.M.; et al. Regulation of chitinase-3-like-1 in T cell elicits Th1 and cytotoxic responses to inhibit lung metastasis. Nat. Commun. 2018, 9, 503. [CrossRef]

27. Lee, C.G.; Da Silva, C.A.; Dela Cruz, C.S.; Ahangari, F.; Ma, B.; Kang, M.J.; He, C.H.; Takyar, S.; Elias, J.A. Role of chitin and chitinase/chitinase-like proteins in inflammation, tissue remodeling, and injury. Annu. Rev. Physiol. 2011, 73, 479-501. [CrossRef]

28. Libreros, S.; Iragavarapu-Charyulu, V. YKL-40/CHI3L1 drives inflammation on the road of tumor progression. J. Leukoc. Biol. 2015, 98, 931-936. [CrossRef] [PubMed]

29. Ngernyuang, N.; Yan, W.; Schwartz, L.M.; Oh, D.; Liu, Y.B.; Chen, H.; Shao, R. A Heparin Binding Motif Rich in Arginine and Lysine is the Functional Domain of YKL-40. Neoplasia 2018, 20, 182-192. [CrossRef]

30. Shao, R.; Taylor, S.L.; Oh, D.S.; Schwartz, L.M. Vascular heterogeneity and targeting: The role of YKL-40 in glioblastoma vascularization. Oncotarget 2015, 6, 40507-40518. [CrossRef]

31. Yeo, I.J.; Lee, C.K.; Han, S.B.; Yun, J.; Hong, J.T. Roles of chitinase 3-like 1 in the development of cancer, neurodegenerative diseases, and inflammatory diseases. Pharmacol. Ther. 2019, 203, 107394. [CrossRef] [PubMed] 
32. Zhao, T.; Su, Z.; Li, Y.; Zhang, X.; You, Q. Chitinase-3 like-protein-1 function and its role in diseases. Signal. Transduct. Target. Ther. 2020, 5, 201. [CrossRef]

33. Benn, M.; Nordestgaard, B.G.; Jensen, G.B.; Tybjaerg-Hansen, A. Improving prediction of ischemic cardiovascular disease in the general population using apolipoprotein B: The Copenhagen City Heart Study. Arterioscler. Thromb. Vasc. Biol. 2007, 27, 661-670. [CrossRef] [PubMed]

34. Park, H.S.; Lee, H.S.; Park, J.S.; Park, J.S.; Lee, D.K.; Lee, S.J.; Yoon, D.S.; Lee, M.G.; Jeung, H.C. Prognostic Scoring Index for Patients with Metastatic Pancreatic Adenocarcinoma. Cancer Res. Treat. 2016, 48, 1253-1263. [CrossRef]

35. Salmiheimo, A.; Mustonen, H.; Stenman, U.H.; Puolakkainen, P.; Kemppainen, E.; Seppanen, H.; Haglund, C. Systemic Inflammatory Response and Elevated Tumour Markers Predict Worse Survival in Resectable Pancreatic Ductal Adenocarcinoma. PLoS ONE 2016, 11, e0163064. [CrossRef]

36. Tempero, M.A.; Uchida, E.; Takasaki, H.; Burnett, D.A.; Steplewski, Z.; Pour, P.M. Relationship of carbohydrate antigen 19-9 and Lewis antigens in pancreatic cancer. Cancer Res. 1987, 47, 5501-5503. 